\title{
FINITE ELEMENT CALCULATIONS AND EXPERIMENTAL VERIFICATION OF THE UNSTEADY POTENTIAL FLOW IN A CENTRIFUGAL VOLUTE PUMP
}

\author{
R. BADIE* AND J. B. JONKER \\ Faculty of Mechanical Engineering, University of Twente, Enschede, Netherlands \\ AND \\ R. A. VAN DEN BRAEMBUSSCHE \\ Von Karman Institute, Sint-Genesius-Rode, Belgium
}

\begin{abstract}
SUMMARY
In this paper we present a finite-element-based method for the calculation of the unsteady potential flow in rotor/stator configurations. A numerical algorithm was developed to calculate the two-dimensional flow through a centrifugal volute pump, taking into account the width variation of the volute in the axial direction and the vortex wakes downstream of the impeller blades by a linearized vortex distribution. The pressure field was obtained from the unsteady Bernoulli equation, with the entire configuration of the pump being included in the calculations. For that purpose the computational domain was split into a region containing the rotor and one containing the stationary parts, each region being treated in a different co-ordinate system. The corresponding finite element grids are matched by an interface consisting of connect elements which move with time. The method is applied to a laboratory centrifugal pump set up at the Von Karman Institute for Fluid Dynamics, which made an experimental validation possible. The total head of the pump and the velocity and pressure fields were computed and analysed for various mass flows. The agreement with the experimental data was satisfactory. The deviation was largest at low mass flow, the maximum deviation in the velocity around the impeller being 10 per cent. The overall behaviour of the pump could be well predicted.
\end{abstract}

KEY wORDS Impeller/volute interaction Unsteady flow Linearized vortex model

\section{INTRODUCTION}

Owing to rotor/stator interaction in centrifugal pumps, many unsteady flow phenomena occur that have great impact on the performance of such machines. A considerable number of these phenomena are related to potential flow interactions (see e.g. Reference 1). Since theoretical calculations of the flow have often been performed by isolating one of the two parts of the pump, one cannot obtain an adequate grasp of the unsteady potential flow features. Encouraged by the successful results of unsteady aerofoil theory, some authors started considering the rotor/stator interaction by using singularity methods applied to axial pumps and fans (see e.g. References 2 and 3) and to centrifugal pumps. ${ }^{4.5}$ For a comprehensive review of these works the reader is referred to References 6 and 7 . Only a few calculations, however, treated centrifugal volute pumps

- Present address: Philips Research, Eindhoven, Netherlands.

CCC 0271-2091/94/241083-20

(C) 1994 by John Wiley \& Sons, Ltd.

Received January 1994

Revised June 1994 
by taking the entire configuration of the pump into account. Morfiadakis et al. ${ }^{8}$ developed a numerical code based on the panel method for simulating the unsteady potential flow in a centrifugal volute pump. The two parts of the pump were considered together and the calculations were compared with experimental results. The increase in the volute width in the axial direction was not included in the calculations, however, which made a comparison with test results very difficult. Furthermore, a very limited number of results could be presented. Recently Croba et al. ${ }^{9}$ applied the finite volume method for the calculation of $2 \mathrm{D}$ unsteady and viscous flow. They used a multidomain overlapping grid technique in order to match the flow fields calculated within the impeller and the fixed volute. Miner et al. ${ }^{10}$ used the finite element method for the calculation of the flow and recently ${ }^{11}$ the forces in a laboratory pump. The rotation of the impeller in the volute was simulated by using steady state solutions with the rotor at various angular positions. However, the partial time derivative of the velocity potential for the calculation of the pressure field, which has a significant influence on the flow quantities under off-design conditions, was neglected.

In this paper we put forward the development of a finite-element-based algorithm to calculate the fully unsteady potential flow in a centrifugal volute pump, taking into account the transient terms and including the vortex sheets downstream of the blades by using a linearized vortex distribution model. The method allows for a variation in the volute width in the axial direction. The computational domain is separated into two regions. The region that contains the impeller is described in a rotating co-ordinate system, while the other region is considered in a stationary co-ordinate system. The corresponding finite element meshes are matched by a sliding interface. The algorithm has been used for the calculation of the flow in the blade-to-blade plane of a pump at the Von Karman Institute for Fluid Dynamics in Belgium. Laser Doppler velocimetry (LDV) and pressure measurements have been performed, enabling an experimental verification of the calculations.

\section{GOVERNING EQUATIONS}

In a centrifugal pump we consider the two-dimensional flow of an incompressible fluid whose absolute velocity vector field is irrotational and we define a time-dependent potential $\phi$ as

$$
\nabla \phi=\mathbf{v}
$$

When this potential exists, it satisfies at each instant the elliptic type of equation

$$
\nabla \cdot(b \nabla \phi)=0
$$

where $b$ is a function which represents the dimensional variation in the direction perpendicular to the plane. This function is necessary since the width of the casing in pumps usually changes in this direction. Equation (2) can be solved with appropriate boundary conditions, after which the potential flow is determined uniquely at a given time. The pressure field can then be obtained from the unsteady Bernoulli equation

$$
\frac{\partial \phi}{\partial t}+\frac{1}{2}|\nabla \phi|^{2}+\frac{p}{\rho}=c(t) .
$$

where $p$ is the pressure, $\rho$ is the density, $t$ is the time and $c$ is the Bernoulli constant. A careful examination of this equation shows that the energy transfer in a pump (i.e. change in total pressure) is attributable to the time derivative of the defined potential. 
The unsteadiness of the flow is introduced through the boundary conditions at the impeller blades, which rotate with respect to the volute casing with an angular velocity $\Omega$. The above-mentioned equation can be transformed to the rotating system by using the identity (see also Reference 12)

$$
\frac{\partial_{\mathrm{R}}}{\partial t}=\frac{\partial}{\partial t}+(\mathbf{\Omega} \times \mathbf{r}) \cdot \nabla
$$

where $\partial_{\mathbf{R}} / \partial t$ is used to denote the time derivative operator with respect to the rotating co-ordinate system. Then equation (3) for the potential, referred to the rotating co-ordinate system, becomes

$$
\frac{\partial_{\mathbf{R}} \phi}{\partial t}-(\boldsymbol{\Omega} \times \mathbf{r}) \cdot \nabla \phi+\frac{p}{\rho}+\frac{1}{2}|\nabla \phi|^{2}=c(t),
$$

\section{LINEARIZED VORTEX DISTRIBUTION DOWNSTREAM OF A THIN IMPELLER BLADE}

From elementary fluid dynamics it is known that the circulation $\Gamma$ of the potential flow around an aerofoil and its wake is a conserved quantity, i.e.

$$
\frac{\mathrm{D} \Gamma}{\mathrm{D} t}=0
$$

When an impeller is rotating in the casing of a centrifugal pump, the circulation around the blades (considered as thin aerofoils) is changing continuously with time according to the unsteady Kutta condition. Equation (6) shows that this change is accompanied by vortex shedding downstream of the blades. In potential flows circulation and vortex shedding are introduced by potential discontinuities on suitable slit lines and wake curves (which are mathematical cuts) in the computational domain (see e.g. Reference 13). To find the equations governing the potential jumps on the wake curves, we apply a linearized model.

Consider a blade with a vortex wake (extending from the trailing edge) which is thin enough to be treated as a curve with two sides: the upper side and the lower side. On this curve the potential jump must be such that the pressure field is continuous everywhere in the fluid. We introduce the following symbols: $\phi_{u}$, the limit of the velocity potential when the wake (or cut) is approached from the upper side of the curve; $\phi_{1}$, the limit of the velocity potential when the wake (or cut) is approached from the lower side of the curve. From the Bernoulli equation (3) it follows that

$$
\frac{\partial}{\partial t}\left(\phi_{1}-\phi_{u}\right)=-\frac{1}{2}\left(\left|\nabla \phi_{1}\right|^{2}-\left|\nabla \phi_{u}\right|^{2}\right)
$$

To apply a linearized wake model, the potential is divided into two subpotentials (representing a mainstream and a perturbation on it),

$$
\phi=\bar{\phi}+\tilde{\phi} \quad(|\nabla \tilde{\phi}| \ll|\nabla \bar{\phi}|)
$$

where $\tilde{\phi}$ is the perturbation caused by the existence of a vortex sheet and $\bar{\phi}$ is the potential due 
to the mainstream (obtained from a quasi-unsteady anlaysis). Using this relation and equation (7), requiring continuity of the pressure across the vortex sheet, we then have

$$
\frac{\partial}{\partial t}\left(\tilde{\phi}_{1}-\tilde{\phi}_{\mathrm{u}}\right)+|\nabla \bar{\phi}|_{\mathrm{s}} \frac{\partial}{\partial s}\left(\tilde{\phi}_{1}-\tilde{\phi}_{\mathrm{u}}\right)=0
$$

where $s$ is the co-ordinate along the wake curve. The above equation determines the vortex distribution along the wake, i.e. the potential jump as a function of time and space.

\subsection{Vortex representation for a blade}

In order to solve the problem formulated in the previous section, a vortex model of the continuous vortex sheet is introduced by a vortex wake curve consisting of vortex elements as shown in Figure 1.

The length of each vortex element is $\Delta s=U_{s} \Delta t$, where $U_{s}$ is the component of the averaged mainstream velocity along the element and $\Delta t$ is the corresponding time step. The strength of the latest vortex element at the trailing edge (i.e. the first wake element downstream of a blade), $\Gamma_{w}^{k}$, is equal to the vorticity shed during the time interval $(k-1) \Delta t<t<k \Delta t$; thus

$$
\Gamma_{w}^{k}=\int_{(k-1) \Delta t}^{k \Delta t} \gamma_{w}^{k} U \mathrm{~d} t,
$$

where $\gamma_{w}^{k}$ is the vortex distribution of element $k$, defined as

$$
\gamma_{w}^{k}=\frac{\partial}{\partial s}\left(\tilde{\phi}_{\mathbf{u}}^{k}-\tilde{\phi}_{1}^{k}\right)
$$

For each segment $\gamma_{w}$ is taken as constant, resulting in linear distribution of the velocity potential jump over the elements.

At each time step newly formed vortices are shed in the wake curve and are moved away with the main flow as described by equation (9). These vortices are added to the already existing wakes. This means that it is possible to fix the path of the vortices if the mainstream is calculated in advance. This is done by a quasi-unsteady calculation. Furthermore, only the part of the vortex wake near the trailing edge is considered, which has the most significant effect on the

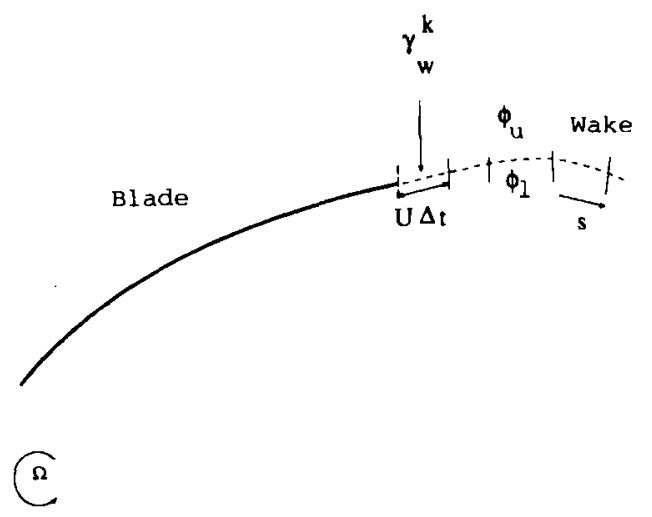

Figure 1. Vortex representation for a blade 
determination of the Kutta condition (the length of this part for the pump model will be given in Section 5). For a more theoretical validation and the adequacy of this model the reader is referred to Reference 14.

In this way the jump in the velocity potential on the wakes is represented by a piecewise linear and continuous function $f_{i}$ for the wake belonging to blade number $i$. This function is dependent on time and the co-ordinates of the points on the wake curves and must satisfy the relation given in equation (9) for the potential jump.

\section{MODELLING OF ROTOR/STATOR INTERACTION}

For the numerical calculation of the partial time derivative of the velocity potential in equation (3) it is necessary that the values of the potential are known at points with the same co-ordinates for different time steps. In a finite element approach this would lead to problems due to moving boundaries if the computational domain were to be regarded from one co-ordinate system. The convenient approach is therefore to split the computational domain into two parts, one part fixed to the stator and the other part rotating with the impeller. The interface between the two parts is introduced as two coinciding circles (a cut in the computational domain that we call a sliding cut from now on) corresponding to the two regions already mentioned. The nodal points (which are distributed equidistantly) on these two circles are connected to each other for a specific time step (i.e. the solution and the derived quantities at these nodal points are mutually related). However, this connection has to change its configuration with time according to the displacement of the rotor with respect to the stator.

Figure 2 illustrates the above-mentioned idea. Two blocks are shown, each in a different system. These blocks are a periodic part of a rotor/stator configuration i.e. a $(1 / n)$ th part of a configuration where the rotor and the stator have each $n$ blades. They are shown in an $(r, \theta)$
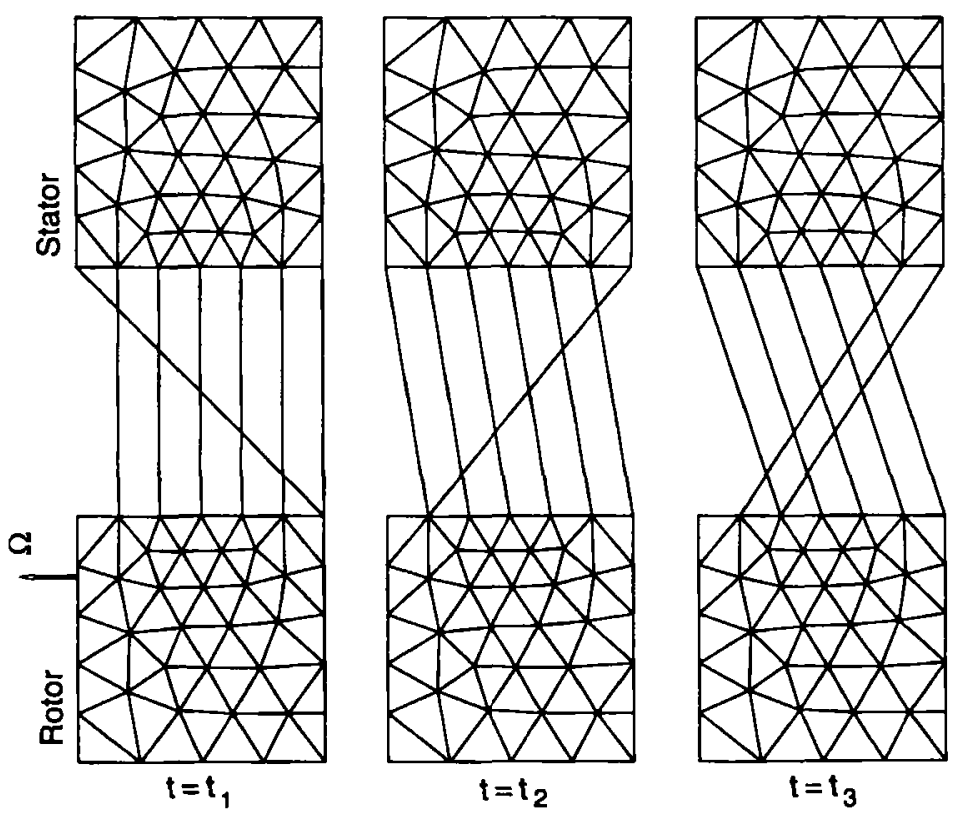

Figure 2. Use of sliding cut 
co-ordinate system, which means that one block is in fact a wedge (or a V-form). For convenience the two blocks have been drawn apart (in reality they touch each other). A connection between the nodal points of the interface (i.e. the sliding cut) is shown by straight lines. The nodal points of the periodic boundaries are connected to each other as well. Thus in some cases more than two points are connected to each other. To define all the connections in a unique way, the starting point is as shown for $t=t_{1}$. At another instant, say $t=t_{2}$, this configuration has to be changed accordingly as shown in the figure. A connection to a point outside the blocks can be avoided by using a point in the domain at which, according to the periodicity, the physical quantities (such as velocity and pressure) have the same value. For more details the reader is referred to Reference 15.

The time derivative of the velocity potential can be calculated by taking at each nodal point the values of the potential before and after a mesh step (one element shift at the sliding interface). If $n_{\mathrm{e}}$ is the total number of elements along the sliding cut, then the time difference associated with a mesh step is defined as

$$
\Delta t=\frac{2 \pi}{\Omega n_{e}} .
$$

The time derivative can then be calculated as a space derivative using the central difference scheme

$$
\frac{\partial \phi}{\partial t}=\frac{\phi_{m+1}-\phi_{m-1}}{2 \Delta t}+O\left((\Delta t)^{2}\right)
$$

where the index $m$ is used to denote the mesh step number.

\section{COMPUTATIONAL DOMAIN AND BOUNDARY CONDITIONS}

Figure 3 shows the computational domain of the flow. The blades and the volute are logarithmic spirals, the dimensions of which are given later in Section 7. The rotor is equipped with $n$ blades

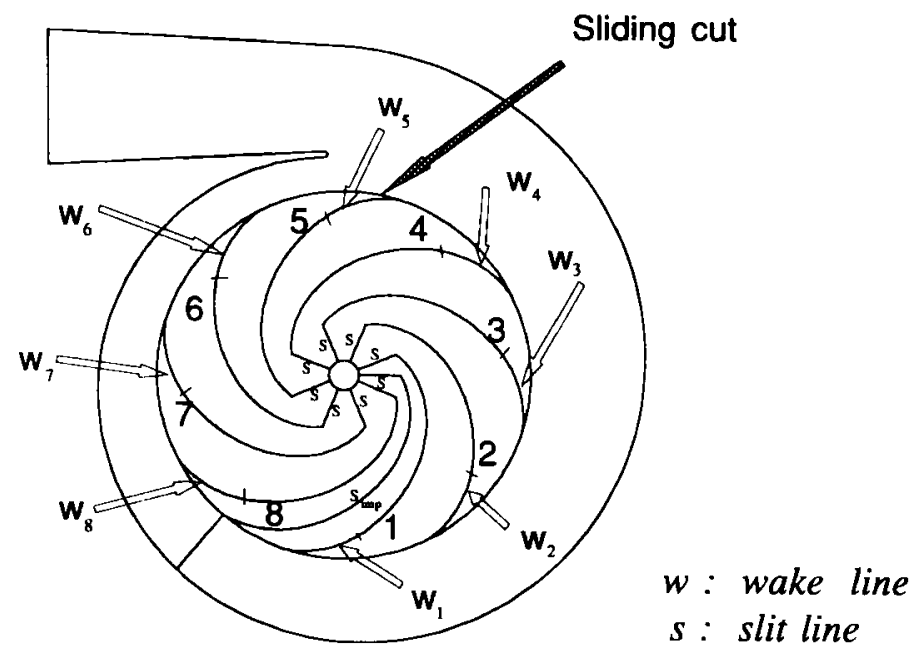

Figure 3. Computational domain 
(in the figure there are eight blades) without thickness. Although the algorithm is capable of handling blades with thickness (as long as they end in a cusp at the trailing edge), we have opted for this simplification, since the camber line of profiles already gives a good deal of relevant data concerning the pump performance, as known from wing theories. Neglecting the blade thickness does have some adverse consequences, e.g. for the velocity near the leading edge and for the calculation of the lateral force on the rotor. These subjects are discussed further in Reference 14. From the trailing edge of each blade a wake curve, denoted by $w_{i}$, extends up to the sliding cut. For the boundary conditions on the solid body, inlet and outlet of the machine the usual conditions are imposed, i.e. at the surface of the solid body the relative normal velocity is equal to zero, while at the inlet and outlet the flow discharge is prescribed. Since the domain is multiply connected, extra conditions will be needed which are related to the circulation of the flow around the blades and wakes. These circulations are realized by introducing potential jumps on slit lines (in the figure denoted by s) and wake vortex curves (denoted by $w_{i}$ in the figure).

The boundary conditions which must be satisfied at each time are

$$
\begin{aligned}
\frac{\partial \phi}{\partial n} & =0 \quad \text { on the stationary parts, } \\
\frac{\partial \phi}{\partial n} & = \pm \Omega r \cos \beta \quad \text { on the rotor blades, } \\
b \frac{\partial \phi}{\partial n} & =\frac{Q}{l_{\text {inlet }}} \text { at the inlet, } \\
b \frac{\partial \phi}{\partial n} & =\frac{-Q}{l_{\text {outlet }}} \quad \text { at the outlet, } \\
\phi_{\mathrm{u}}-\phi_{\mathrm{l}} & =\Gamma_{i} \quad \text { across slit line } i, \\
\phi_{\mathrm{u}}-\phi_{1} & =\Gamma_{\text {out }} \quad \text { across slit line } s_{\text {imp }}, \\
\phi_{\mathrm{u}}-\phi_{1} & =f_{i} \quad \text { on wake number } i \\
\phi & =0 \quad \text { at a point of the inlet, }
\end{aligned}
$$

where $l_{\text {inlet }}$ and $l_{\text {oullet }}$ are the lengths of the inlet and outlet respectively, $n$ is the normal direction, $\beta$ is the blade angle, $\Omega$ is the angular speed and $Q$ is the volume flow. $\Gamma_{i}$ and $f_{i}$ have to be determined such that the Kutta condition is satisfied (see next section). Note that $\Gamma_{i}$ is the circulation of blade $i$ and its wake and $f_{i}$ is the distribution of the velocity potential jump for that wake. The sign for the boundary condition on the rotor blades is dependent upon the side of the blade considered.

An extra cut from the inlet to the outer boundary must be introduced $\left(s_{\text {imp }}\right)$ in order to impose $\Gamma_{\text {out }}$. This circulation can be determined from the relation

$$
\Gamma_{\text {out }}=\Gamma_{\text {in }}+\sum_{1}^{n} \Gamma_{i}
$$

where $\Gamma_{\text {in }}$ represents the pre-rotation at the inlet. 


\section{KUTTA CONDITION}

To determine the strength of the newly formed vortices, satisfying the Kutta condition at each instant, the linear property of the operators can be used. For each time step the velocity potential is divided into $n+1$ parts,

$$
\phi=\phi_{0}+\sum_{i=1}^{n} \Gamma_{W_{i}}^{k} \phi_{i}^{k}
$$

where $\phi_{0}$ is the potential due to blade rotation, flow discharge and the blade and wake circulations developed up to the actual time, excluding the newly formed vortices, $\Gamma_{w_{i}}^{k}$ are $n$ unknown constants to be calculated and $\phi_{i}^{k}$ is the potential due to a linear unit vortex distribution on the latest element of the wake belonging to blade number $i$ (i.e. newly formed unit vortex element). By a unit vortex distribution we imply that this subpotential is solved by imposing a potential jump on the vortex wake which has the value unity at the blade trailing edge and decreases linearly to zero at position $U_{s} \Delta t$. The value of $U_{s}$ is a function of time and space as mentioned earlier. To satisfy the Kutta condition at a blade trailing edge, it is necessary that the following relation holds at each instant:

$$
\left.\frac{\partial \phi}{\partial n}\right|_{\text {tip }}=\Omega r_{\text {tip }} \cos \beta_{\text {tip }},
$$

where the index 'tip' is used to indicate the trailing edge of the blades. Using this condition and solving the subpotentials of equation (15) separately, a linear system of equations is obtained which can be solved as the matrix-vector equation

$$
\left(\begin{array}{ccc}
\left.\frac{\partial \phi_{1}^{k}}{\partial n}\right|_{\text {tip 1 }} & \cdots & \left.\frac{\partial \phi_{n}^{k}}{\partial n}\right|_{\text {tip 1 }} \\
\vdots & & \vdots \\
\left.\frac{\partial \phi_{1}^{k}}{\partial n}\right|_{\text {tipn }} & \cdots & \left.\frac{\partial \phi_{n}^{k}}{\partial n}\right|_{\text {tip } n}
\end{array}\right)\left(\begin{array}{c}
\Gamma_{\mathrm{W} 1}^{k} \\
\vdots \\
\Gamma_{\mathrm{W} n}^{k}
\end{array}\right)+\left(\begin{array}{c}
\left.\frac{\partial \phi_{0}}{\partial n}\right|_{\text {tip 1 }} \\
\vdots \\
\left.\frac{\partial \phi_{0}}{\partial n}\right|_{\text {tip } n}
\end{array}\right)=\left(\begin{array}{c}
\Omega r_{\text {tip } 1} \cos \beta_{\text {tip 1 }} \\
\vdots \\
\Omega r_{\text {tip } n} \cos \beta_{\text {tipn }}
\end{array}\right) .
$$

Then $\Gamma_{\mathrm{W} i}^{k}$ (the unknown constants) are the strength values of the newly formed vortex elements for blade number $i$ such that the Kutta condition is satisfied for the time step considered. The boundary conditions for the total problem as mentioned in the previous section are thus determined at each time step. In this manner the problem is solved (after having completed a quasi-stationary calculation) iteratively by starting from a flow free from vortex wakes. Since we seek a periodic solution in time with the blade passing frequency, the configuration of the vortex distribution must ultimately show the same periodic behaviour. The convergence criterion to be met verifies this periodicity of the flow. The following criterion is used in this paper:

$$
\max |\phi(\vec{x}, t)-\phi(\dot{x}, t-T)|<\varepsilon, . t \in[k T,(k+1) T], \quad \vec{x} \in S, \quad k=2,3, \ldots, m,
$$

where $S$ is the surface of the computational domain, $\varepsilon$ is a user-defined quantity, $T$ is the time length for one pitch period (i.e. $2 \pi / \Omega n$ ) and $m$ is the maximum number of iterations.

The matrix in equation (17) is only dependent on the rotor position. Therefore its elements are stored when calculations for the first pitch rotation are performed and subsequently used for other iterations, which reduces the computation time remarkably. 


\section{TEST FACILITY}

A schematic picture of the experimental set-up is shown in Figure 4. The facility is operated with water and has been specially built for LDV measurements at the Von Karman Institute for Fluid Dynamics in Belgium. ${ }^{16}$ The impeller and casing are made of Plexiglass. The impeller has eight blades with $60^{\circ}$ outlet lean angle and is driven by a variable speed DC motor. The measurements have been performed at an angular rotor speed of $31 \mathrm{rad} \mathrm{s}^{-1}$. The mass flow is measured by a venturi at the impeller inlet and controlled by means of a throttling valve at the outlet. Measurements have been made for low, medium (or optimum) and high mass flows.

The diffuser is instrumented with static pressure tappings positioned around the impeller exit. The main parameters of the pump geometry and operating conditions are given in Table $I$. The symbols used are explained in Figure 5. The LDV measuring system used for the determination of the time-averaged velocities at the impeller outlet is schematically presented in Figure 6. It consists of a laser, a beam splitter, a Bragg cell, lenses and a photodetector. The argon ion laser has a power of $15 \mathrm{~mW}$ with a wavelength of $514.5 \mathrm{~nm}$ and a beam diameter of $1.5 \mathrm{~mm}$. The distance between the two parallel beams is $130 \mathrm{~mm}$ and the optical system can be rotated to measure the tangential and radial velocity components separately. A Bragg cell is used in order to realize a frequency shift between the two laser beams. This instrument is required because of the possible change in sign of the radial velocity components. The two beams are focused by a lens with focal length of $480 \mathrm{~mm}$. The scattered light is focused on a photomultiplier where it is transformed into an electrical signal. The filtered signal enters a counterprocessor system which verifies the quality of the signal and calculates the instantaneous velocity, which is stored on a cassette logger together with the impeller circumferential position at which measurements are taken. 1024 samples are taken for each measurement point between hub and shroud. Subsequently an average and a standard deviation are computed. Latex particles of $1-4 \mu \mathrm{m}$

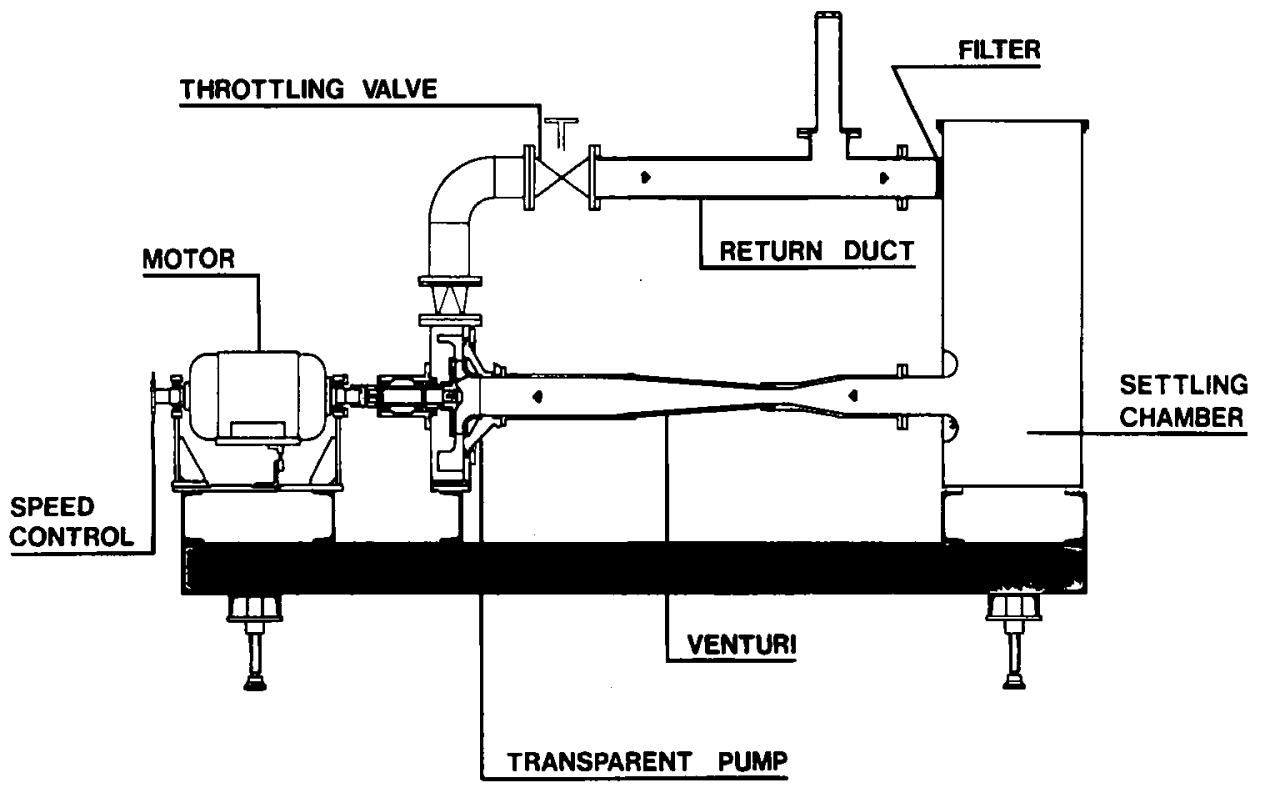

Figure 4. Pump test facility P1 
Table I. Main pump parameters

Volute parameters

Angle of volute spiral Polar co-ordinates of tongue

Radius of spiral's end point Width in axial direction

$\begin{array}{lc}\alpha & 86.235^{\circ} \\ r_{\mathrm{A}} & 0.145 \mathrm{~m} \\ \theta_{\mathrm{A}} & 95^{\circ} \\ r_{\mathrm{C}} & 0.218 \mathrm{~m} \\ b_{\text {vol }} & 0.070 \mathrm{~m}\end{array}$

Operating conditions

Impeller angular speed

Low mass flow

Optimum mass flow

High mass flow

$\begin{array}{ll}\Omega & 31 \mathrm{rad} \mathrm{s}^{-1} \\ Q_{1} & 0.0027 \mathrm{~m}^{3} \mathrm{~s}^{-1}(49 \%) \\ Q_{\mathrm{o}} & 0.0055 \mathrm{~m}^{3} \mathrm{~s}^{-1}(100 \%) \\ Q_{\mathrm{h}} & 0.0067 \mathrm{~m}^{3} \mathrm{~s}^{-1}(122 \%)\end{array}$

Impeller parameters

Number of blades

Blade angle

$\begin{array}{lc}n & 8 \\ \beta & 60 \cdot 332^{\circ} \\ r_{1} & 0.037 \mathrm{~m} \\ r_{2} & 0 \cdot 100 \mathrm{~m} \\ b_{\text {imp }} & 0.020 \mathrm{~m}\end{array}$

Radius of trailing edge

Width in axial direction

$b_{\text {imp }}$

diameter are added to the water; the filter in the return duct takes out all particles larger than $10 \mu \mathrm{m}$.

Only a small part of the measurement error is dependent on the calibration and geometrical parameters of the LDV system. The main sources of error are the non-uniform distribution of particles in the flow and the statistical treatment which is applied to the data measured in an unsteady flow. These errors are very difficult to calculate; they have been estimated by comparing

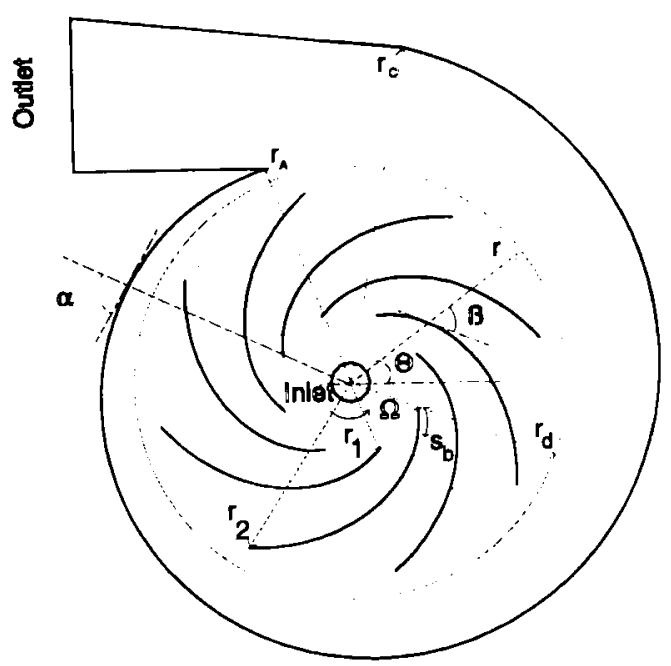

Figure 5. Geometrical data 


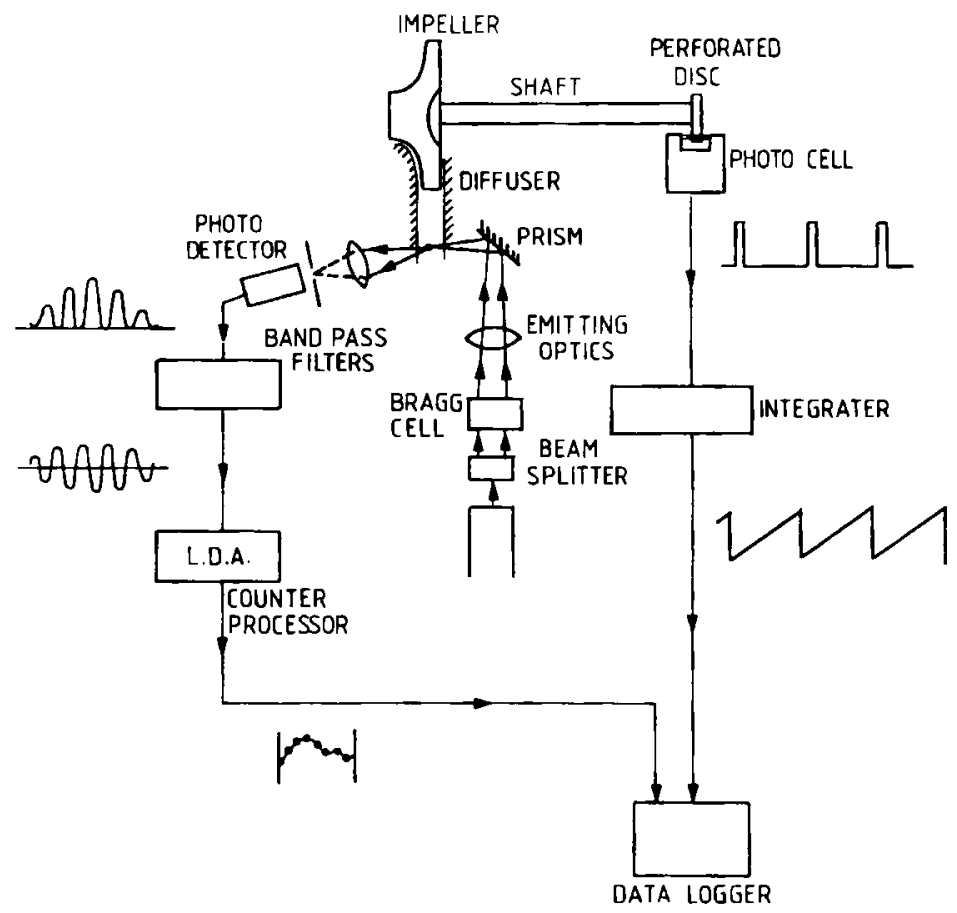

Figure 6. LDV measuring system

the integrated radial velocity with the mass flow measured by the venturi. The error has been found to be between $5 \%$ and $10 \%$. More details on the measuring system can be found in Reference 16.

\section{RESULTS}

Based on the finite element code SEPRAN ${ }^{17}$ an algorithm has been implemented that handles all the previously mentioned manipulations and calculates the potential flow in a rotor/stator configuration. This algorithm has been applied to the previously described centrifugal pump.

The 2D mesh necessary for the finite element calculations is illustrated in Figure 7. To obtain reliable results, the mesh has to satisfy some important requirements. The mesh has to be refined at the boundaries as known from elementary finite element theory. This refinement must increase at sharp edges such as the trailing edge of the blades. This is obvious, since the sharp edges form singularities in a potential flow. The circulation around the blades determines the work of the pump and this is in turn dependent on the Kutta condition. The calculations must therefore be more accurate in the neighbourhood of the trailing edges where the Kutta condition is applied. It is also appropriate to introduce a certain refinement at the leading edge, tongue and in a ring at the impeller outlet for including the width variation of the volute in the third dimension. The choice of an optimum mesh was made after several tests. The mesh shown in Figure 7 has approximately 10,000 triangular linear elements including boundary elements (this amounts to 5000 nodal points approximately). Calculations have been performed with 160 elements on the sliding cut. The physical situation which exists at any instant is precisely repeated when the impeller has moved through one blade pitch. Calculations are therefore presented for one period 


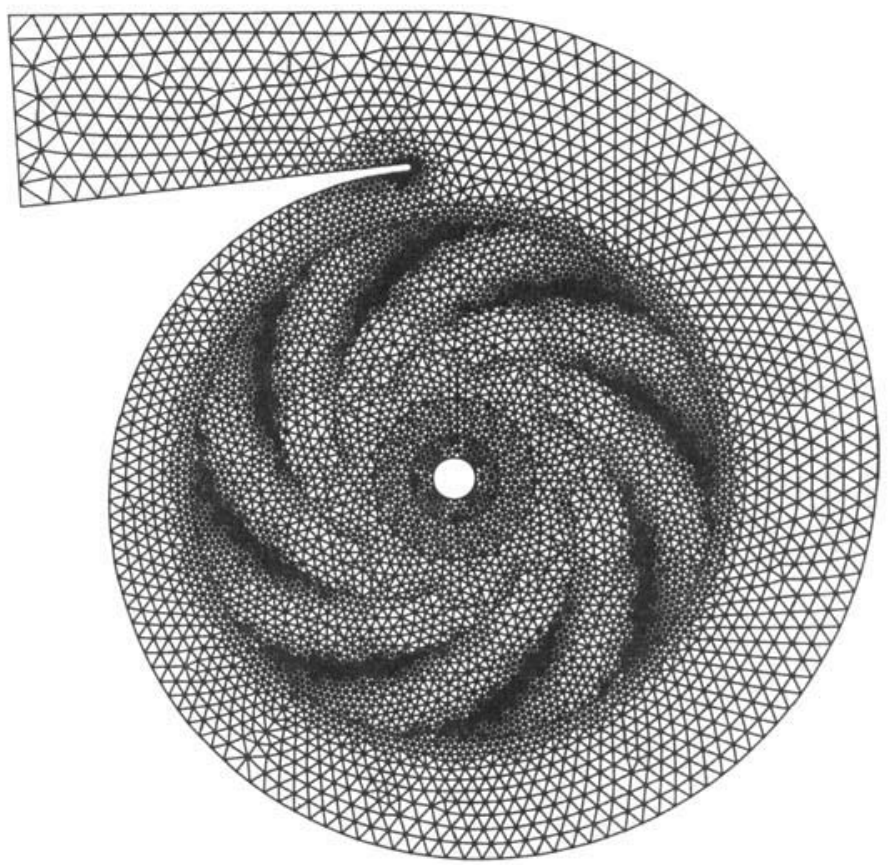

Figure 7. Finite element mesh

only. The numerical data were sampled for 10 different instants corresponding to impeller positions from $0^{\circ}$ to $45^{\circ}$ where the initial configuration (i.e. $0^{\circ}$ ) is as shown in Figure 5.

The computer used was a CONVEX C240. Solving one subpotential with given boundary conditions for the mesh shown in Figure 7 requires an average of $25 \mathrm{~s}$ of CPU time. For an accuracy of $\varepsilon=0.01$ in equation (18) eight pitch iterations (as mentioned above) were necessary (compare the value of $\varepsilon$ with the maximum difference in the velocity potential occurring in the pump, which is of the order of magnitude of unity). This means that after one full rotation of the impeller the required accuracy has been reached.

Figure 8 shows the measured head-capacity characteristic of the pump compared with that obtained from numerical calculations. It is possible to derive the head of the pump either by considering the conservation of the moment of momentum or by integration of the pressure along the blade surfaces. In this case, since we are dealing with blades without thickness, direct numerical integration of the pressure along the blades neglects the leading edge forces that are present as known from wing theory. Calculations considering the conservation of the moment of momentum, however, take these forces into account if a suitable control volume is chosen. Comparison of the results for the head of the pump by the two methods showed that in this particular case the leading edge forces had no significant resulting moment.

It is seen from Figure 8 that the characteristic calculated by the present method is not a straight line as it should be in the case of a free impeller (i.e. not including the volute configuration). Thus it follows that by taking into account the potential flow mismatch between the impeller and the volute (this subject is thoroughly treated in Reference 18), better agreement with the experimental results can be achieved. In this case there is still a noticeable difference between the calculated head and the measured one (approximately $25 \%$ for the nominal point 


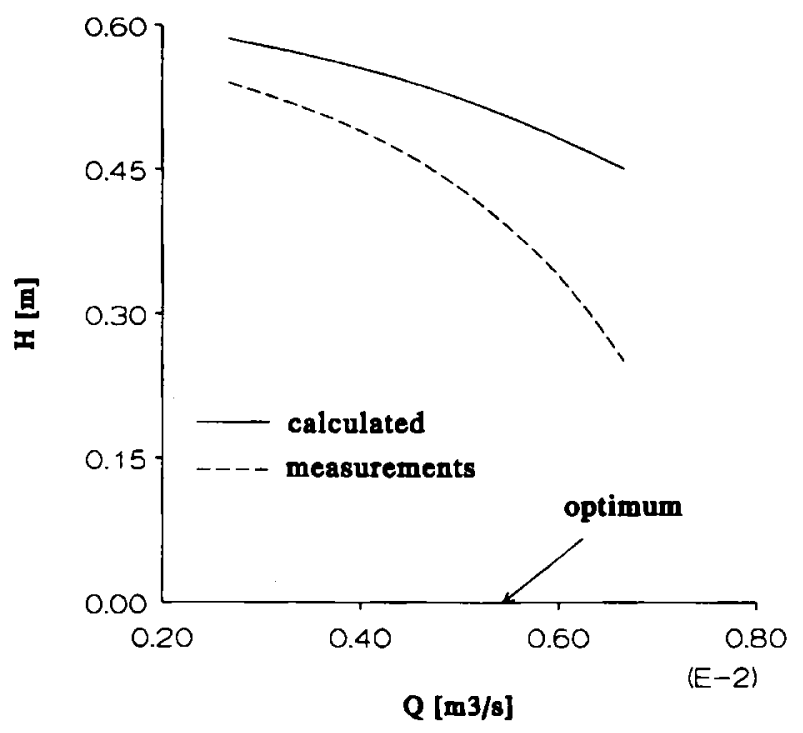

Figure 8. Head-capacity characteristic

of operation). The major friction losses occurring in the pump are due to separation of the flow at the blade leading edge and at the tongue, wall friction losses and three-dimensional viscous flow phenomena in the volute. These losses have an important influence on the pressure rise in the pump even at optimum mass flow (except for the separation at the volute tongue which does not occur at optimum mass flow). The velocity field is, however, less affected by these friction losses (see Reference 15 for further explanation).

The fact that the calculated head is very close to the experimental data at low mass flows can be misleading. One might conclude that the potential flow calculations approach the real flow better in the case of low mass flow than in the case of other mass flows. That such a conclusion is incorrect follows from Figure 9, where the calculated pressure around the impeller is compared with the experimentally obtained pressure measured with respect to the inlet stagnation pressure. The predicted circumferential pressure variation is larger than the experimental one at low mass flow, although the average pressure is well predicted by the calculations. The circumferential variation is, however, better predicted at medium and high mass flows. It is the error in the average pressure value which is increasing with mass flow (see also Reference 14; the pressure variations especially near the volute tongue are very important when studying some physical phenomena such as pump noise; see e.g. Reference 19).

The time-averaged circumferential velocity along a circle around the impeller $(r=0.116 \mathrm{~m})$ is plotted in Figure 10 for low, optimum and high mass flows. The numerical results are compared with experimental data averaged in the axial direction. There are rapid and strong changes calculated in the region near the tongue (at $\theta=95^{\circ}$ ), especially under off-design conditions. In reality these fluctuations will diminish by viscosity effects. Taking the accuracy of the LDV measurements into account, we can observe that the potential calculations approach the test data satisfactorily. The discrepancy is most conspicuous at low mass flow (10\% on average). For optimum and high mass flows the numerical results differ by only $2 \%-5 \%$ from the experimental data.

The relative tangential velocities and the pressure along the blades are important quantities 

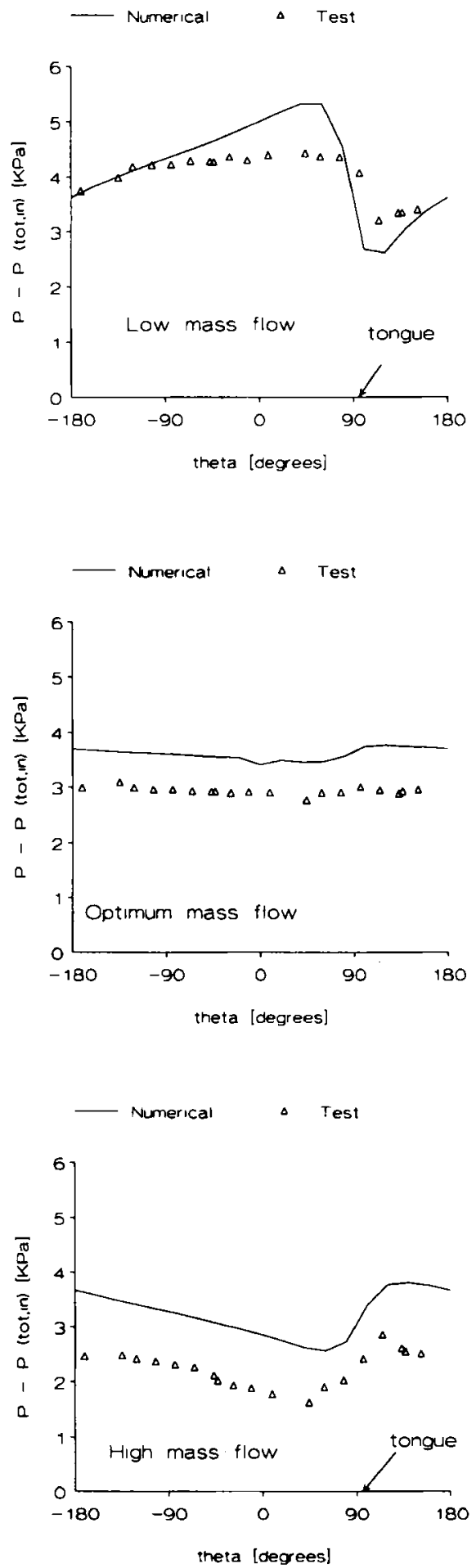

Figure 9. Pressure around the impeller $(r=0.102 \mathrm{~m})$ 

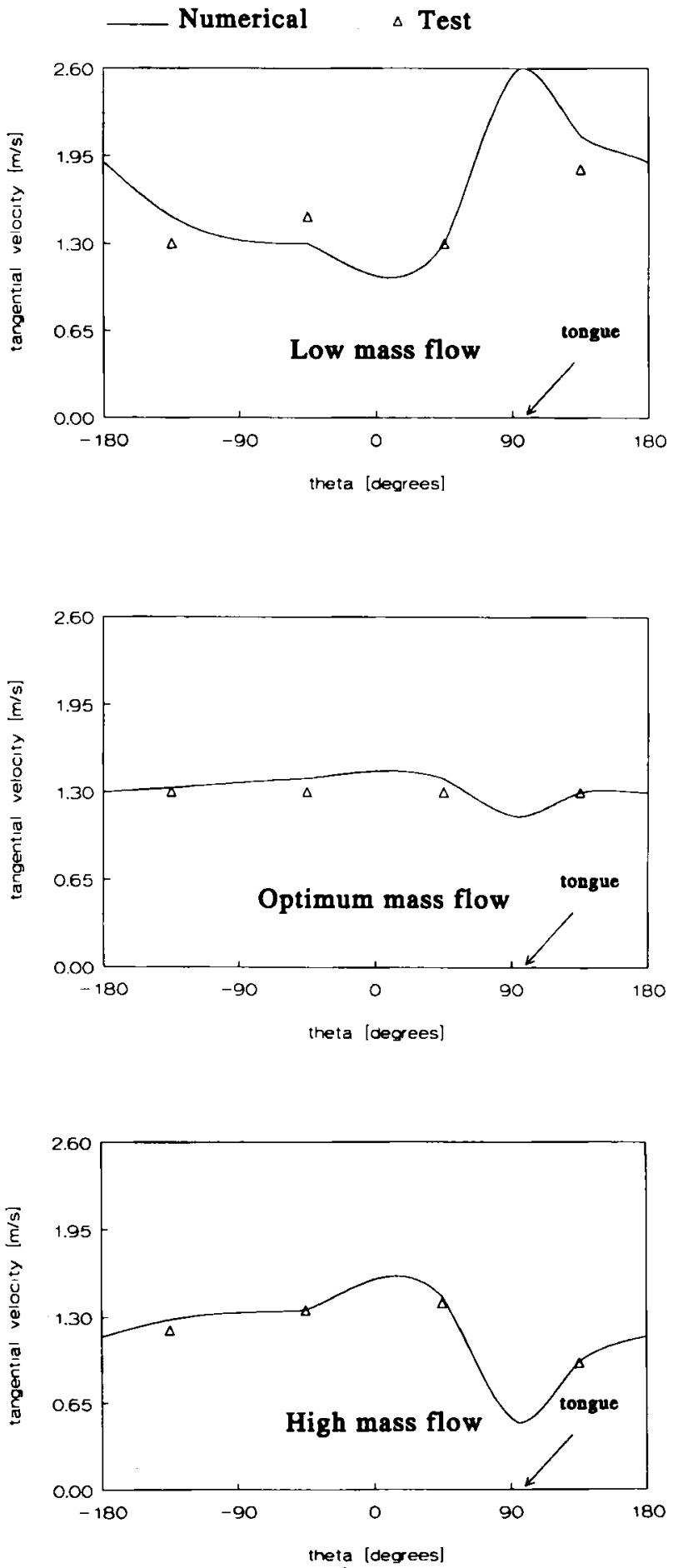

Figure 10. Circumferential velocity around the rotor $(r=0.116 \mathrm{~m})$ 

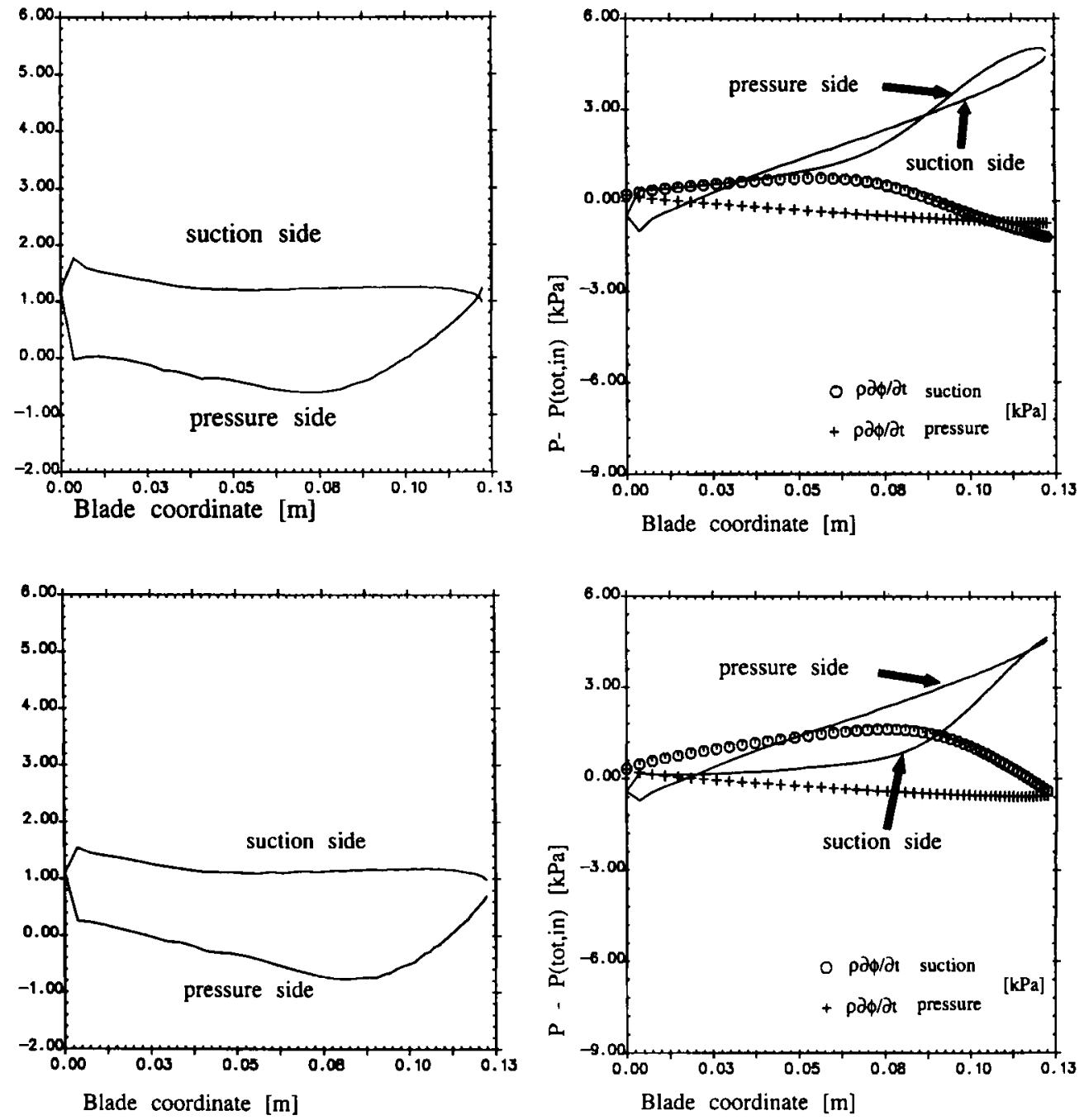

Figure 11. Velocity, pressure and relative time derivative of potential along blade number 4 for low mass flow for two different time steps $(t=0 \mathrm{~s}$ and $t=0.010 \mathrm{~s})$

for characterizing the behaviour of the flow in the impeller. The influence of the volute on these quantities is significant at off-design. Owing to lack of space, we will show these quantities only along one blade for low mass flow at two different times; see Figure 11 (for more results the reader is referred to Reference 14). The quantity $\rho \partial_{R} \phi / \partial t$ is also shown in the diagrams in order to illustrate the importance of this term in the Bernoulli equation. When dealing with a free impeller (i.e. the flow is time-independent in the rotating system), this term is equal to zero. Neglecting this term at off-design is, however, seen to have far-reaching consequences for the blades approaching the tongue.

Strong negative velocities and an adverse pressure gradient are noticeable in the case of low mass flow. It is seen that for a blade approaching the tongue, the pressure on the surface, which is normally the suction surface, exceeds that of the pressure side. These strong deformations of the flow field (with respect to the flow in an isolated impeller) are not tolerated by real fluids 
and the flow is often prone to separation. Basically, such a phenomenon (i.e. separation) is possible on both sides of the blade. Of course, potential flow calculations cease to be valid in regions where separation has occurred.

The absolute velocity vectors in the case of all three mass flows have been plotted in Figure 12. The behaviour of the flow near the tongue agrees with that observed by experiments (see e.g. Reference 20). Notice how the stagnation point moves around the tongue when changing the mass flow. Within the framework of potential theory we define the optimum flow as one for which on one hand there is no mismatch between the rotor and the volute and on the other hand there is no reverse flow at the blade surface. It is interesting to note that the optimum flow calculated numerically in this way (i.e. satisfying both conditions) agreees with the experimental one accurately. This means that the present method can be used to optimize the shape of a volute for a given impeller (or the shape of an impeller for a given volute).

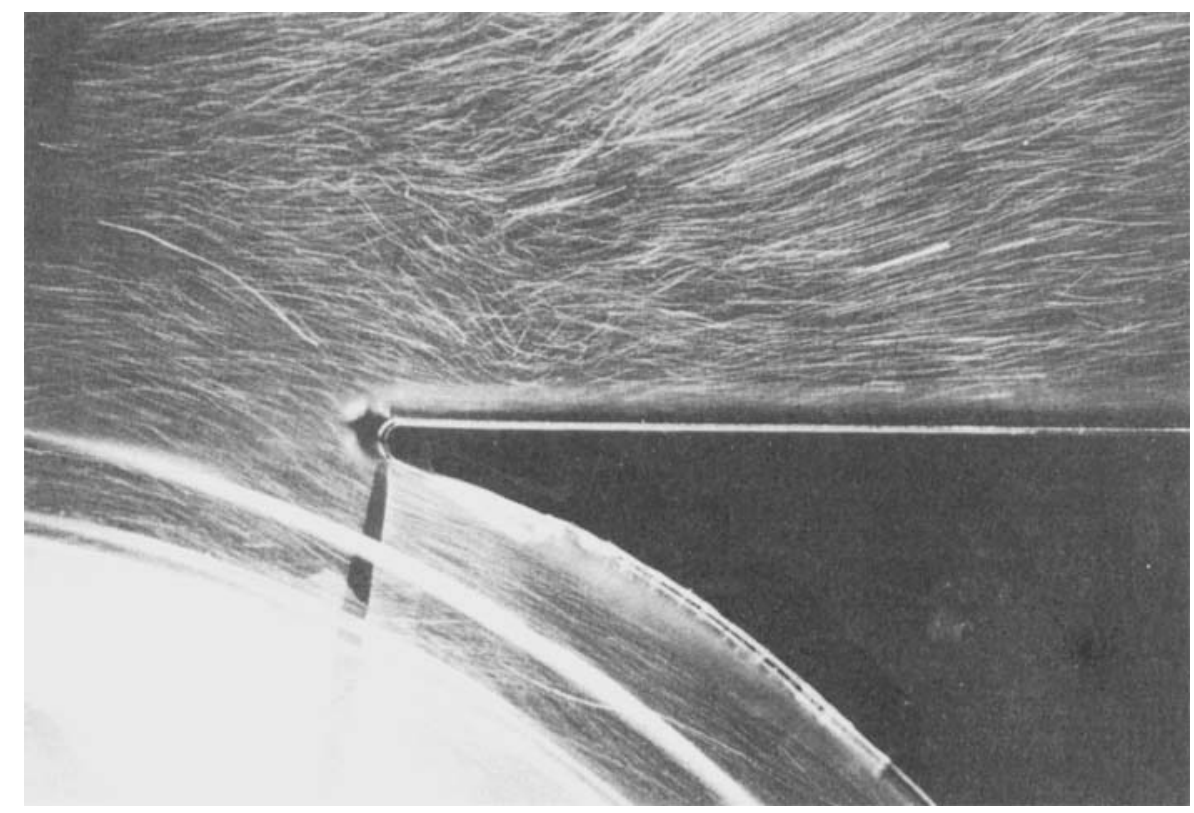

Flow visualization (Von Karman Institute)
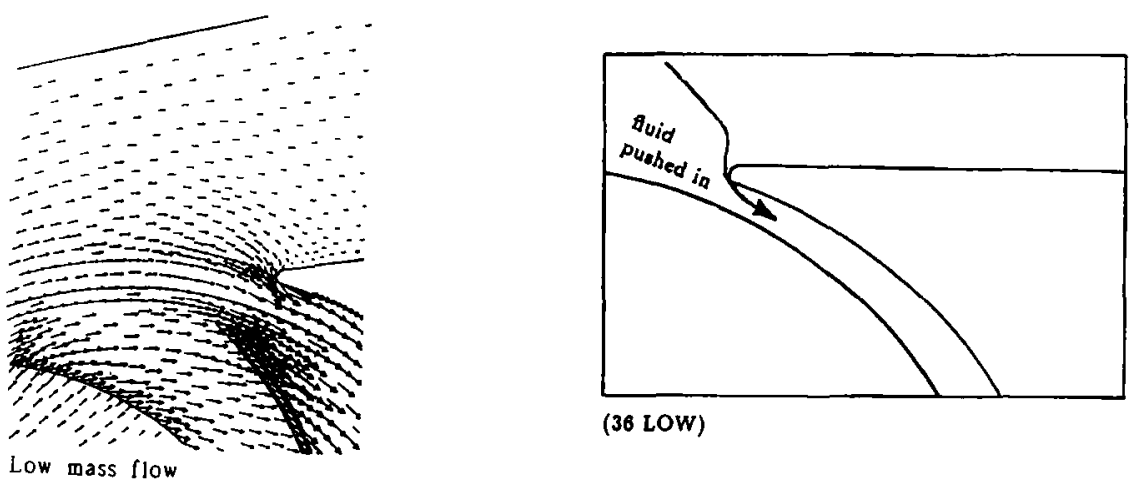

(36 LOW)

Numerical Calculations

Figure 12(a). Flow behaviour near the tongue at low mass flow; comparison of numerical calculations and flow visualization 


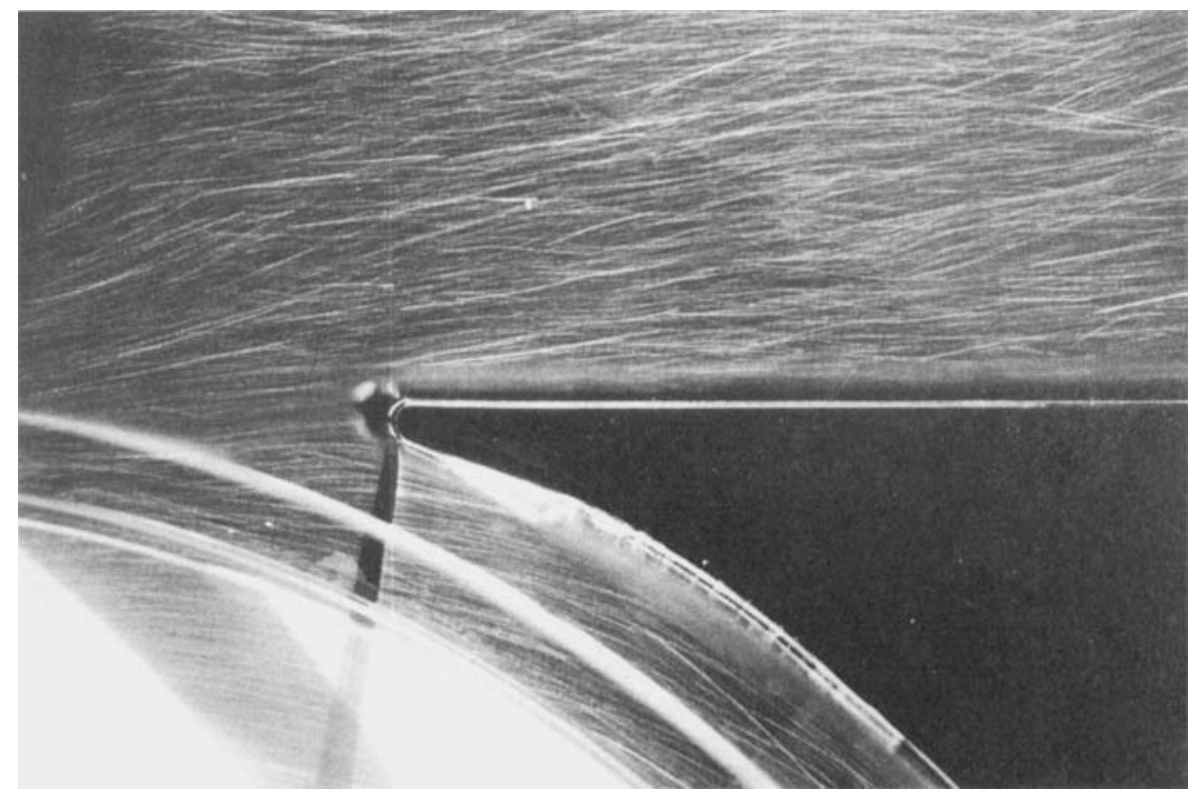

Flow visualization (Von Karman Institute)
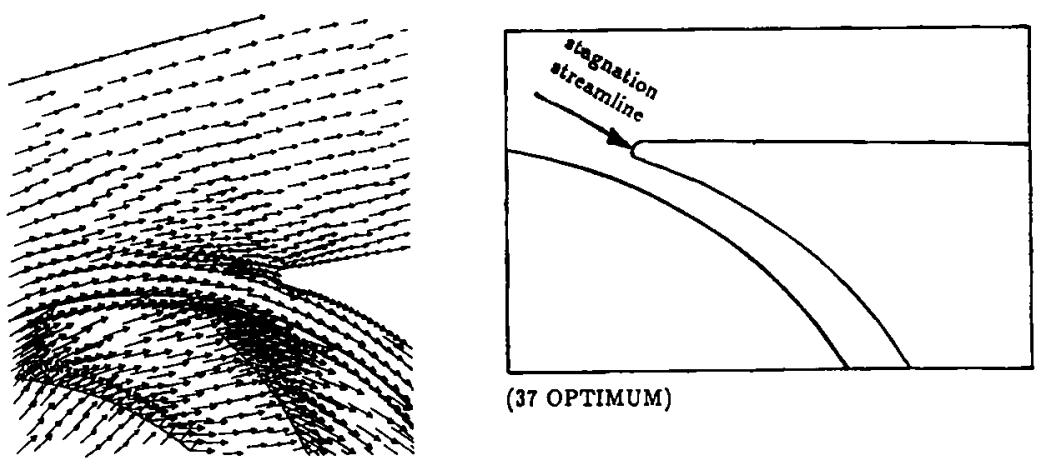

(37 OPTIMUM)

Optunum mass flow

Numerical Calculations

Figure 12(b). Flow behaviour near the tongue at optimum mass flow; comparison of numerical calculations and flow visualization 


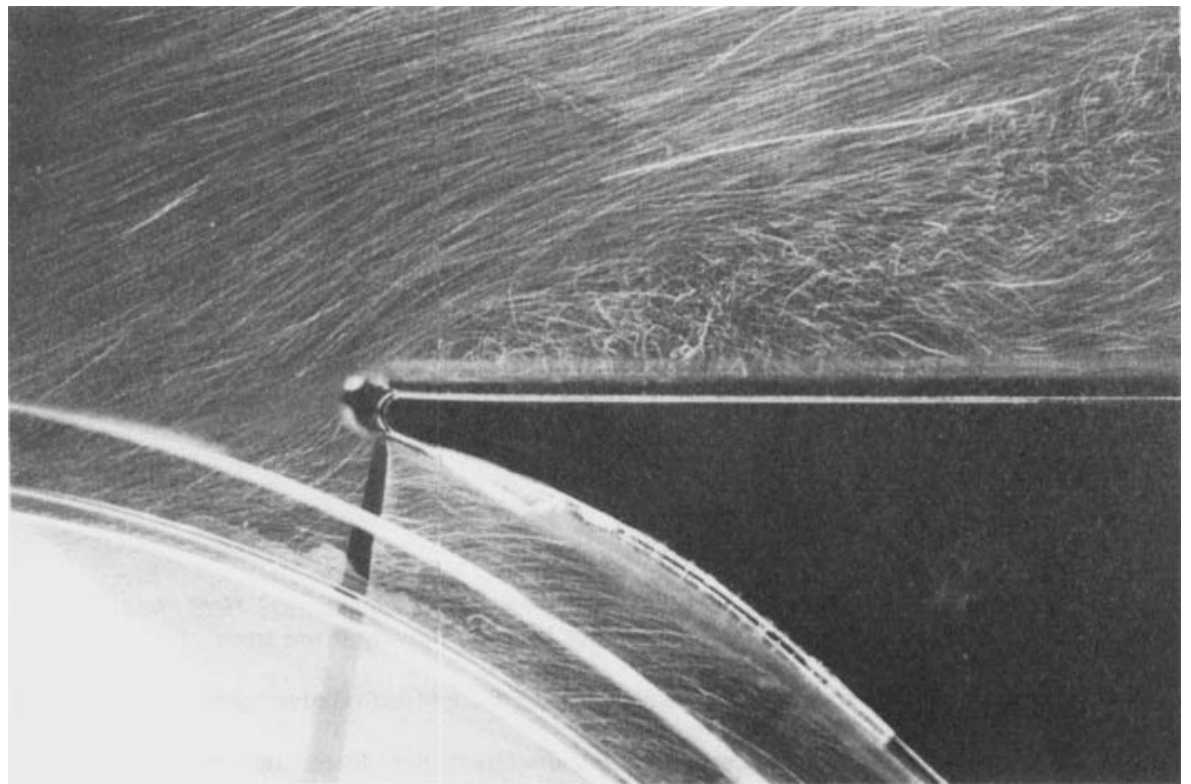

Flow visualization (Von Karman Institute)
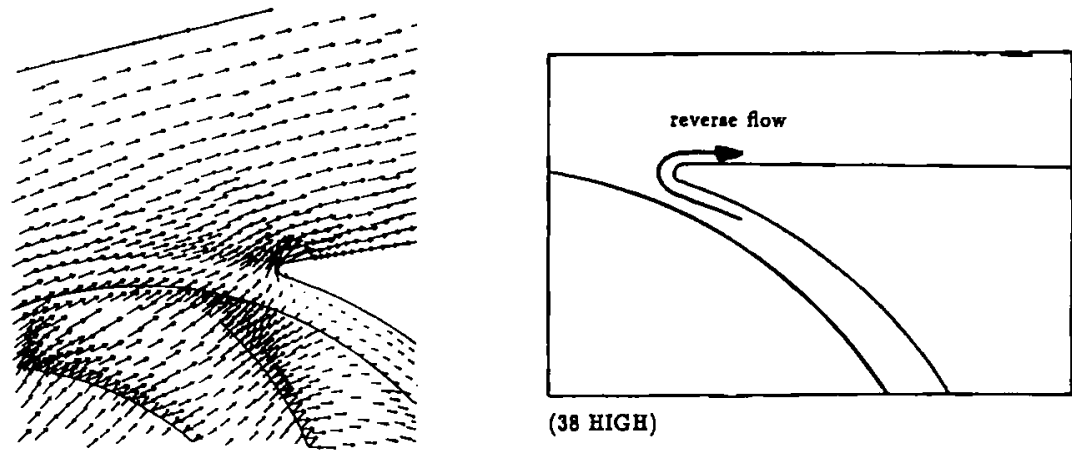

(38 HIGH)

High mass flow

Numerical Calculations

Figure 12(c). Flow behaviour near the tongue at high mass flow; comparison of numerical calculations and flow visualization

\section{CONCLUSIONS}

Application of a finite-element-based algorithm to the flow in a centrifugal volute pump was presented and used for calculation of the unsteady two-dimensional potential flow including wake vortices. The geometries of both parts were modelled together and the time derivative of the velocity potential calculated adequately. The results showed the following.

1. The tangential velocity and the pressure variation in the volute around the impeller agree satisfactorily with the experimental results. The qualitative correspondence was least at below-design flow. The quantitative average deviation from the experimental data for the circumferential velocity around the impeller was approximately $10 \%$ for low mass flow. The discrepancy at medium and high mass flows was $2 \%-5 \%$. 
2. The transient term in the unsteady Bernoulli equation $(\partial \phi / \partial t)$ plays an important part under off-design conditions.

3. It is possible to optimize the shape of a volute for a given impeller and required specifications (or the shape of an impeller for a given volute) by means of the present method. For this optimum configuration there is on one hand no mismatch between the rotor and the volute and on the other hand no reverse flow at the blade surface.

4. The behaviour of the flow near the tongue under design and off-design conditions can be adequately predicted by potential flow calculations.

5. By the present numerical calculations it is possible to include the non-symmetrical character of the flow around the impeller when computing important pump parameters such as the head.

\section{REFERENCES}

1. R. Parker and J. F. Watson, 'Interaction effects between blade rows in turbomachines'. Heat Fluid Flow, 2, 33 (1972).

2. N. H. Kemp and W. R. Sears, 'Aerodynamic interference between moving blade rows', J. Aeronaut. Sci., 20, 585 (1953).

3. D. N. Gorelov, 'Hydrodynamic interaction of cascade of plates during their relative motion', Zh. Prikl. Mekh. Tekh. Fiz., (1), 40 (1974).

4. Y. Tsujimoto, A. J. Acosta and C. E. Brennen, 'Theoretical study of fluid forces on a centrifugal impeller rotating and whirling in a volute', J. Vibr. Acoust., Stress Reliab. Design, 110, 263 (1988).

5. T. lino, 'Potential interactions between a centrifugal impeller and a vaned diffuser, ASME, Winter Annual Reports, Fluid Structure Interactions in Turbomachinery, ASME, New York, 1981, p. 63.

6. E. M. Greitzer, 'Unsteady flow in turbomachines: recent advances and opportunities for control', Proc. Gas Turbine Conf., Tokyo, 1987.

7. M. F. Platzer, 'Unsteady flows in turbomachinery: a review of current developments', in Ashely et al. (eds), Unsteady Aerodynamics: AGARD Symp., Ottawa, 1977, Vol. 33, p. 1.

8. E. E. Morfiadakis, S. G. Voutsinas and D. E. Papantonis, 'Unsteady flow calculation in a radial flow centrifugal pump with spiral casing', Int. $j$. numer. methods fluids, 12, 895 (1991).

9. D. Croba, J. L. Kueny, F. Hureau and J. Kermarec, 'Numerical and experimental unsteady flow analysis in centrifugal pumps. Impeller and volute interaction', Proc. 1st Int. Symp. on Pump Noise and Vibrations, 1993, p. 111.

10. S. M. Miner, R. D. Flack and P. E. Allaire, 'Two-dimensional flow anlaysis of a laboratory centrifugal pump', ASME Paper 90-GT-50, 1990.

11. S. M. Miner, R. D. Flack and P. Trevisan, 'Potential flow anlaysis of the forces on a laboratory centrifugal pump impeller', ASME Paper 92-GT-285, 1992.

12. D. T. Greenwood, Principles of Dynamics, Prentice-Hall, Englewood Cliffs, NJ, 1965.

13. D. H. Norrie and G. de Vries, The Finite Element Method, American Press, New York, 1973.

14. R. Badie, 'Analysis of unsteady potential flows in a centrifugal pump', Doctor's Thesis, University of Twente, 1993.

15. R. Badie, J. B. Jonker and T. G. van Essen, 'Calculations on the time-dependent potential flow in a centrifugal pump', ASME Paper 92-GT-151, 1991.

16. R. A. van den Braembussche and $M$. Th. Sideris, 'Influence of circumferential exit pressure distortion on the flow in an impeller and diffuser'. Société Hydrodynamique de France, Quest. 1, Rapport 12, 1989.

17. A. Segal and N. Praagman, SEPRAN Programmers Guide, Ingenieursbureau Sepra, Leidschendam, 1989.

18. H. W. Iversen, R. E. Rolling and J. J. Carlson, 'Volute pressure distribution, radial force on the impeller and volute mixing losses of a radial flow centrifugal pump', ASME J. Eng. Power, 82, 136 (1960).

19. S. Chu. R. Dong and J. Kantz, 'The effect of blade-tongue interaction on the flow structure, pressure fluctuations and noise within a centrifugal pump', Proc. Ist Int. Symp. on Pump Noise and Vibrations, 1993, p. 13.

20. B. Eck, Fans (transl. by Azad and Scott), Pergamon, Oxford, 1973. 